\title{
Multi-joint coordination of vertical arm movement
}

\author{
Ajay Seth,' John J McPhee, ${ }^{2}$ Marcus G Pandy' \\ 'Department of Biomedical Engineering, The University of Texas at Austin, Austin, TX, USA; ${ }^{2}$ Department of Systems \\ Design Engineering, University of Waterloo, Waterloo, Ontario, Canada
}

\begin{abstract}
A model of the human arm was developed to study coordination of multi-joint movement in the vertical plane. The arm was represented as a two-segment, two-degree of freedom dynamic system with net muscle torques acting at the shoulder and elbow. Kinematic data were collected from a subject who performed unrestrained vertical movements with only the initial and final hand elevations prescribed. Movements were performed with and without a hand-held load. The method of computed torques was implemented to obtain net muscle torques, which enables position and velocity feedback to be used to estimate joint angular accelerations that produce a more stable simulation of arm movement. The model simulation was then used to calculate the contributions of the net muscle torques, gravitational torques and velocity-interaction torques to the angular accelerations of the shoulder and elbow and also to the vertical acceleration of the hand. The net muscle torques and gravity were the prime movers of the arm. The velocity-dependent effects contributed little to the dynamics of arm movement and were, in fact, insignificant when the hand was loaded. The muscles of the shoulder and elbow acted synergistically to elevate the arm in the sagittal plane. The hand was accelerated upward by the elbow first, until the point of maximum elbow flexion, after which the shoulder became the prime mover. Gravity acted consistently to accelerate the hand downward. Coordination was notably invariant to changes in external load. Some compensation for load was observed in the control, and these differences were attributed mainly to an increase in system inertia.
\end{abstract}

Keywords: upper limb, multi-joint coordination, induced acceleration, interaction torque, muscle torque

\section{Introduction}

Voluntary human arm movements have been studied extensively in order to understand how movement control is organised and, more specifically, to identify rules that might explain how the central nervous system (CNS) coordinates multi-joint movement under varying conditions of speed and load (Flash and Hogan 1985; Uno et al 1989; Gottlieb et al 1996, 1997; Galloway and Koshland 2002). One recurring theme in these studies is the role that interaction torques play when the CNS must control movement of multiple joints simultaneously. Hollerbach and Flash (1982) defined two types of interaction torques in multi-joint movement: inertial torques, which are torques proportional to joint acceleration; and velocity torques, which are torques proportional to the product of joint velocities. If interaction torques are significant during normal movement, they will influence the trajectory of the limb, and the CNS must then provide the controls necessary to compensate for their presence. In studying the dynamics of two-joint arm movement, Hollerbach and Flash (1982) found that interaction torques are indeed significant over a range of movement speeds and movement paths. They also found that inertial torques are generally more significant than velocity torques during normal human arm movement.

In this study, a slightly different approach is taken in studying multi-joint coordination of human arm movement. Rather than evaluate the contributions of the interaction torques during normal arm movement, the contributions of the net muscle (or joint) torques to the acceleration of each joint at each instant during the movement was calculated. This approach affords additional insight into coordination of multi-joint arm movement because the results not only take into account the presence of interaction torques, they also specify how each net muscle torque contributes to the acceleration of the hand at each point along its trajectory. At each instant during the movement, each net muscle torque accelerates all the joints simultaneously because of a phenomenon known as dynamic coupling. Specifically, for any multi-joint dynamical system modelled using joint (relative) coordinates, the mass matrix is non-diagonal,

Correspondence: Ajay Seth, Department of Biomedical Engineering, The University of Texas at Austin, I University Station C0800, Austin, TX 787 I2, USA; tel + I $5 \mid 2232$ |4 |2; fax + I 5 |2 47| 06|6; email aseth@mail.utexas.edu 
which guarantees that its inverse is also non-diagonal. Thus, in solving for the joint accelerations by pre-multiplying by the inverse of the mass matrix, all net muscle torques are coupled into all joint angular accelerations (Zajac and Gordon 1989; Pandy et al 1990; Pandy 2001).

Coordination of unrestrained vertical arm movement was studied using a simple two-degree of freedom, planar model of the human arm. Vertical arm movement was chosen to determine the effect of gravitational torques on multi-joint coordination during normal arm movement. Movements were performed unloaded and with a hand-held weight so that the effect of load on movement kinematics and dynamics could also be studied. Three specific questions were addressed:

1. What are the relative contributions of the net muscle torques, gravitational torques and velocity-interaction torques during unrestrained vertical arm movement?

2. How are movements of the shoulder and elbow coordinated in elevating the arm?

3. How is arm movement coordination affected by the presence of a hand-held load?

\section{Methods}

Marker kinematics were captured from a test subject performing multiple trials of an unrestrained vertical arm movement. In all trials, the subject was seated comfortably with his back resting firmly against the high back of a chair to minimise translations of the shoulder joint. At the beginning of the task, the shoulder and elbow were extended, and the arm hung comfortably alongside the subject's torso. The subject was then instructed to move his hand to a maximum comfortable height, without abducting the shoulder. No constraints were placed on the initial or final position of the hand or on the time taken to complete the movement. After some practice, the start and end elevations of the hand were recorded, and these elevations were then prescribed during data collection. The task was performed both unloaded and with a 5-kg mass held in the hand. The same start and end elevations of the hand were prescribed for both the unloaded and loaded movements.

Data were collected for five trials of each condition (loaded and unloaded). Marker kinematics were converted to segmental rotations to minimise error in a least-squares sense (Biryukova et al 2000). The motion was then projected to the sagittal plane for each trial. Each trial was 'clipped', such that the data represented a continuous segment of motion above a certain threshold, and its time span yielded the performance time. Each trial was normalised by the performance time. The means and standard deviations of the joint angles were generated for each condition (loaded and unloaded), and these data were then rescaled to the average performance time for the two conditions, respectively.

A multibody dynamics approach was used to generate the equations of motion for a planar two-segment model of the human arm. The modelling software package DynaFlex (http://real.uwaterloo.ca/ dynaflex) was used to generate the dynamical equations of motion. This approach uses linear graph theory and the principles of mechanics to automatically generate the kinematic and dynamic equations for a multibody system, given only a system description file (Shi and McPhee 2002). DynaFlex is written in the Maple $^{\mathrm{TM}}$ computer algebra language, which means that the equations are generated in very efficient symbolic form that can be viewed, manipulated or exported as optimised C, Fortran or MATLAB ${ }^{\circledR}$ code.

Shown in Figure 1A is a schematic diagram of the upper limb. Rectangles represent rigid bodies, and the attached circles represent body-fixed reference frames defining points of interconnection. The torso was assumed to remain stationary and treated as the inertial reference frame (ground). The shoulder and elbow were modelled as ideal revolute (pin or hinge) joints, along with a corresponding joint torque, and the hand was welded to the wrist. Gravity acted through the centre of mass of each body (hashed circles in Figure 1A), and the wrist joint location was used to track the motion of the end effector (hand).

The linear graph corresponding to the link-segment model of Figure 1A is shown in Figure 1B. Nodes represent body-fixed reference frames, while edges are physical elements that act between these frames. Edges $m_{1}, m_{2}$ and $m_{3}$ represent the mass-based properties (inertias and weights) of the three rigid bodies, while edges $r_{4}-r_{7}$ correspond to kinematic transformations between frames (nodes) fixed to the same body. Edges $h_{8}$ and $h_{9}$ represent the two revolute joints corresponding to $\theta_{1}$ and $\theta_{2}$, including the corresponding muscle-generated torques as edges $T_{11}$ and $\mathrm{T}_{12}$. Applying principles of linear graph theory and elementary mechanics to Figure 1B, the governing kinematic and dynamic equations can be generated in a variety of forms to suit the modeller's needs. Linear graph theory offers many advantages over conventional approaches to multibody dynamics; a complete review is given by Shi and McPhee (2000). 


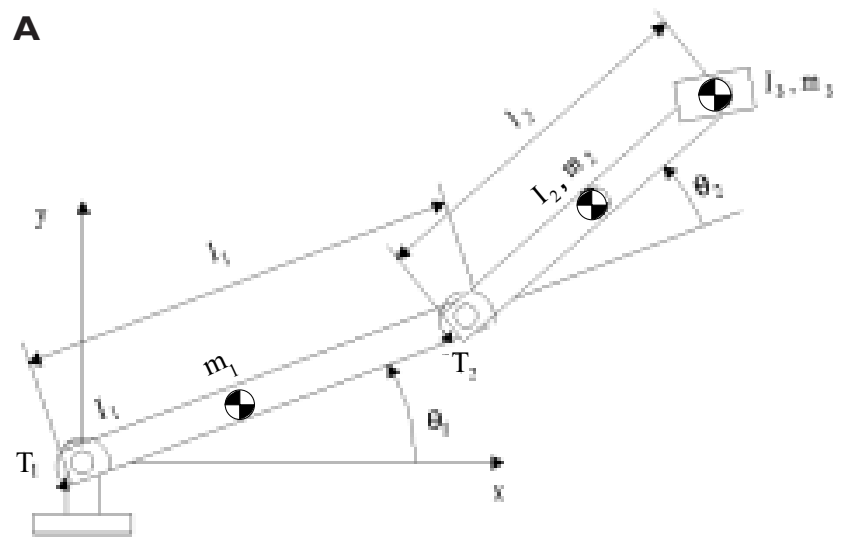

B

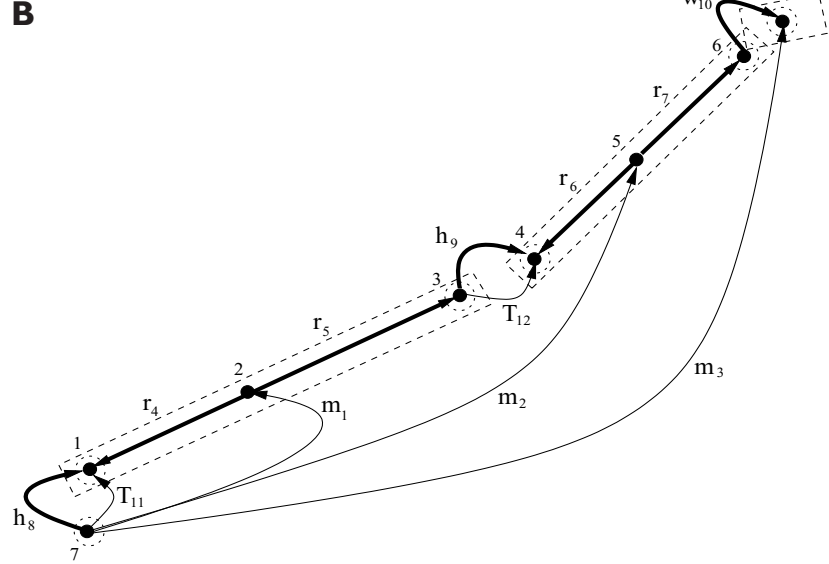

Figure I Schematic diagram (A) and linear graph (B) of the two-segment planar dynamical model of the human arm. Model parameters such as masses $(m)$, moments of inertia $(I)$, segment lengths $(l)$ and fixed distances $(r)$, as well as the remaining symbols, are defined in Appendix I.The shoulder and elbow joint angles $\left(\theta_{1}\right.$ and $\theta_{2}$, respectively) are the model's two degrees of freedom and are controlled by two joint torques $\left(T_{1}\right.$ and $\left.T_{2}\right)$.

From the graph representation, an input file can be generated that encapsulates the linear graph in Figure 1B and the parameters associated with the individual components, eg mass, length and moment of inertia (see Appendix 1). DynaFlex reads this model file and formulates the kinematic and dynamic equations for the corresponding system. For the two-segment system shown in Figure 1, there is one joint coordinate, $\theta$, for each degree of freedom. DynaFlex was used to form the minimal set of two ordinary differential equations governing the dynamics of this system, thus:

$$
\mathbf{M}(\boldsymbol{\theta}) \ddot{\boldsymbol{\theta}}=\mathbf{V}(\boldsymbol{\theta}, \dot{\boldsymbol{\theta}})-\mathbf{G}(\boldsymbol{\theta})+\mathbf{T}
$$

where $\mathbf{M}$ is the symmetric mass matrix; $\boldsymbol{\theta}=\left[\theta_{1}, \theta_{2}\right]^{\mathrm{T}} ; \mathbf{T}$ is a column matrix of joint torques, which represents the net effect of all muscles acting at each joint; $\mathbf{G}$ is a column matrix of torques due to gravitational forces; and $\mathbf{V}$ is a column matrix of quadratic velocity terms (centripetal and Coriolis). Details of equation (1) are given in Appendix 1. Mass, inertial and maximum torque values for the upper- limb model were obtained from the literature (Kingma et al 1996; Garner and Pandy 2001). The contributions of ligaments and other connective tissue to the joint torques were considered part of the net muscle torques computed from the inverse dynamics analysis.

Net muscle torques acting at the shoulder and elbow joints were computed by a nonlinear optimal feedback control method using feedback linearisation. The mean experimental joint trajectories were differentiated twice to yield the necessary input estimates of the desired joint velocities and accelerations. Equation (1) was then solved for the direct computed torques as follows:

$$
\mathbf{T}=\mathbf{M}(\boldsymbol{\theta}) \ddot{\boldsymbol{\theta}}-\mathbf{V}(\boldsymbol{\theta}, \dot{\theta})-\mathbf{G}(\boldsymbol{\theta})
$$

However, if $\ddot{\boldsymbol{\theta}}$ is obtained from double differentiation of nonideal data, it will not be consistent with the behaviour of an ideal model. In addition, taking into account physical differences in model and subject parameters, this will lead to inverse dynamics torques that will not drive the model through the observed kinematics in a forward simulation, since errors accumulate throughout the integration and cause the model kinematics to diverge (Runge et al 1995; Risher at al 1997).

To address this problem, we substituted a control variable such that $\ddot{\boldsymbol{\theta}}=\boldsymbol{v}$. The control $\boldsymbol{v}$ was defined so that it had stable and fast dynamics as a function of an approximation of the observed accelerations ( $\ddot{\boldsymbol{\theta}}_{\text {obs }}$ from double-differentiating the angular positions) and the errors with respect to the observed angular positions and velocities of the joints (Slotine and Li 1991; Vincent and Grantham 1997). Thus,

$$
\boldsymbol{v}=\ddot{\boldsymbol{\theta}}_{\mathrm{obs}}-2 \lambda\left(\dot{\boldsymbol{\theta}}-\dot{\boldsymbol{\theta}}_{\mathrm{obs}}\right)-\lambda^{2}\left(\boldsymbol{\theta}-\boldsymbol{\theta}_{\mathrm{obs}}\right)
$$

where $\lambda$ is the magnitude of the real left-hand plane (stable) poles of the controller $\boldsymbol{v}$. This substitution transformed the solution for the joint torques as follows:

$$
\mathbf{T}=\mathbf{M}(\boldsymbol{\theta}) \boldsymbol{v}-\mathbf{V}(\boldsymbol{\theta}, \dot{\boldsymbol{\theta}})-\mathbf{G}(\boldsymbol{\theta})
$$

The observed angular kinematics were obtained from the marker positions through the data collection process to produce $\boldsymbol{\theta}_{\text {obs }}$, and differentiated to yield $\dot{\boldsymbol{\theta}}_{\mathrm{obs}}$. We used the double differentiation of $\boldsymbol{\theta}_{\text {obs }}$ as an initial estimate of the actual model joint accelerations, $\ddot{\boldsymbol{\theta}}$, and termed it $\ddot{\boldsymbol{\theta}}_{\text {obs }}$, which we expect to be noisy. Thus, the control variable, $\boldsymbol{v}$, was determined by tuning $\ddot{\theta}_{\text {obs }}$ by the feedback of the error between the actual model states and the observed (or desired) kinematics. In this way, $v$ represents a better estimate of the joint accelerations required for the calculation of the joint torques. Note, in particular, that the 
instantaneous model states (angular positions, $\boldsymbol{\theta}$, and angular velocities, $\dot{\boldsymbol{\theta}}$ ) now determine the instantaneous control $\boldsymbol{v}$.

With the instantaneous control defined, we solved for the applied joint torques, $\mathbf{T}$, substituted these values back into equation (1), and then integrated the equations of motion to determine the state at the subsequent instant; this procedure also yielded the control vector, $v$. By integrating across the performance time, beginning with the model state equal to the observed angular position and velocity, the complete torque profile necessary to track the observed kinematics was obtained. This method produced continuous time torques that, when used to drive the model in a forward dynamic simulation, satisfied the input kinematics for the aggregate of each task's trials.

The angular accelerations of the joints were calculated by solving equation (1) given the state, $(\boldsymbol{\theta}, \dot{\boldsymbol{\theta}})$, and the computed joint torques, $\mathbf{T}$, thus:

$$
\ddot{\boldsymbol{\theta}}=\mathbf{M}^{-1}(\mathbf{V}(\boldsymbol{\theta}, \dot{\boldsymbol{\theta}})-\mathbf{G}(\boldsymbol{\theta})+\mathbf{T})
$$

The contributions of the applied net joint torques, $\mathbf{T}$, the gravitational torques, $\mathbf{G}$, and velocity-dependent torques, $\mathbf{V}$, to the joint angular accelerations were computed by setting all other contributions to zero, in turn, and then computing $\ddot{\boldsymbol{\theta}}$.

The induced accelerations of the hand were determined from the kinematic constraint equations of the hand position with respect to the joint (shoulder and elbow) motion. From this relationship, the acceleration of the hand was expressed as a function of the joint states, $\boldsymbol{\theta}$ and $\dot{\boldsymbol{\theta}}$, and the secondorder derivatives, $\ddot{\boldsymbol{\theta}}$. These relations are derived in Appendix 1. The vertical acceleration of the hand is given by the following kinematic equation:

$$
\begin{aligned}
\ddot{y}= & {\left[\begin{array}{ll}
\left(l_{1} \cos \left(\theta_{1}\right)+l_{2} \cos \left(\theta_{1}+\theta_{2}\right)\right) & l_{2} \cos \left(\theta_{1}+\theta_{2}\right)
\end{array}\right] \ddot{\boldsymbol{\theta}} } \\
& -\left[\begin{array}{ll}
\left(l_{1} \sin \left(\theta_{1}\right)+l_{2} \sin \left(\theta_{1}+\theta_{2}\right)\right) & l_{2} \sin \left(\theta_{1}+\theta_{2}\right)
\end{array}\right] \dot{\boldsymbol{\theta}}^{2} \\
& -2 l_{2} \sin \left(\theta_{1}+\theta_{2}\right) \dot{\theta}_{1} \dot{\theta}_{2}
\end{aligned}
$$

The joint angular accelerations were eliminated by substituting equation (5) into equation (6), thus:

$$
\begin{aligned}
\ddot{y}= & {\left[\begin{array}{ll}
\left(l_{1} \cos \left(\theta_{1}\right)+l_{2} \cos \left(\theta_{1}+\theta_{2}\right)\right) & l_{2} \cos \left(\theta_{1}+\theta_{2}\right)
\end{array}\right] } \\
& \left\{\mathbf{M}^{-1}(\mathbf{V}(\boldsymbol{\theta}, \dot{\boldsymbol{\theta}})-\mathbf{G}(\boldsymbol{\theta})+\mathbf{T})\right\} \\
& -\left[\begin{array}{ll}
\left(l_{1} \sin \left(\theta_{1}\right)+l_{2} \sin \left(\theta_{1}+\theta_{2}\right)\right) & l_{2} \sin \left(\theta_{1}+\theta_{2}\right)
\end{array}\right] \dot{\boldsymbol{\theta}}^{2} \\
& -2 l_{2} \sin \left(\theta_{1}+\theta_{2}\right) \dot{\theta}_{1} \dot{\theta}_{2}
\end{aligned}
$$

Equation (7) provides a final symbolic expression for the vertical acceleration of the hand (in global Cartesian coordinates) as a function of the state $(\boldsymbol{\theta}, \dot{\boldsymbol{\theta}})$ and the applied
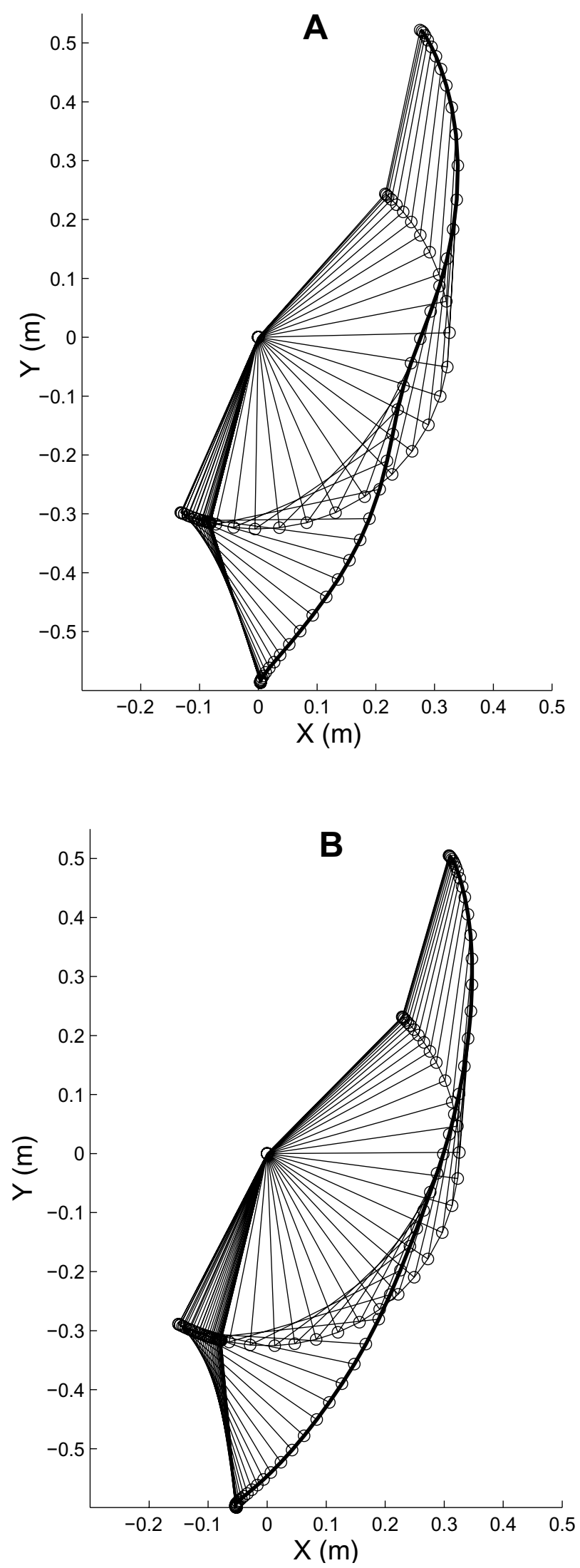

Figure 2 Stick-figure diagram showing the absolute positions of the upper arm and forearm recorded for unloaded (A) and loaded (B) unrestrained vertical arm movement. The loaded movement was performed with a $5-\mathrm{kg}$ weight held in the hand. 

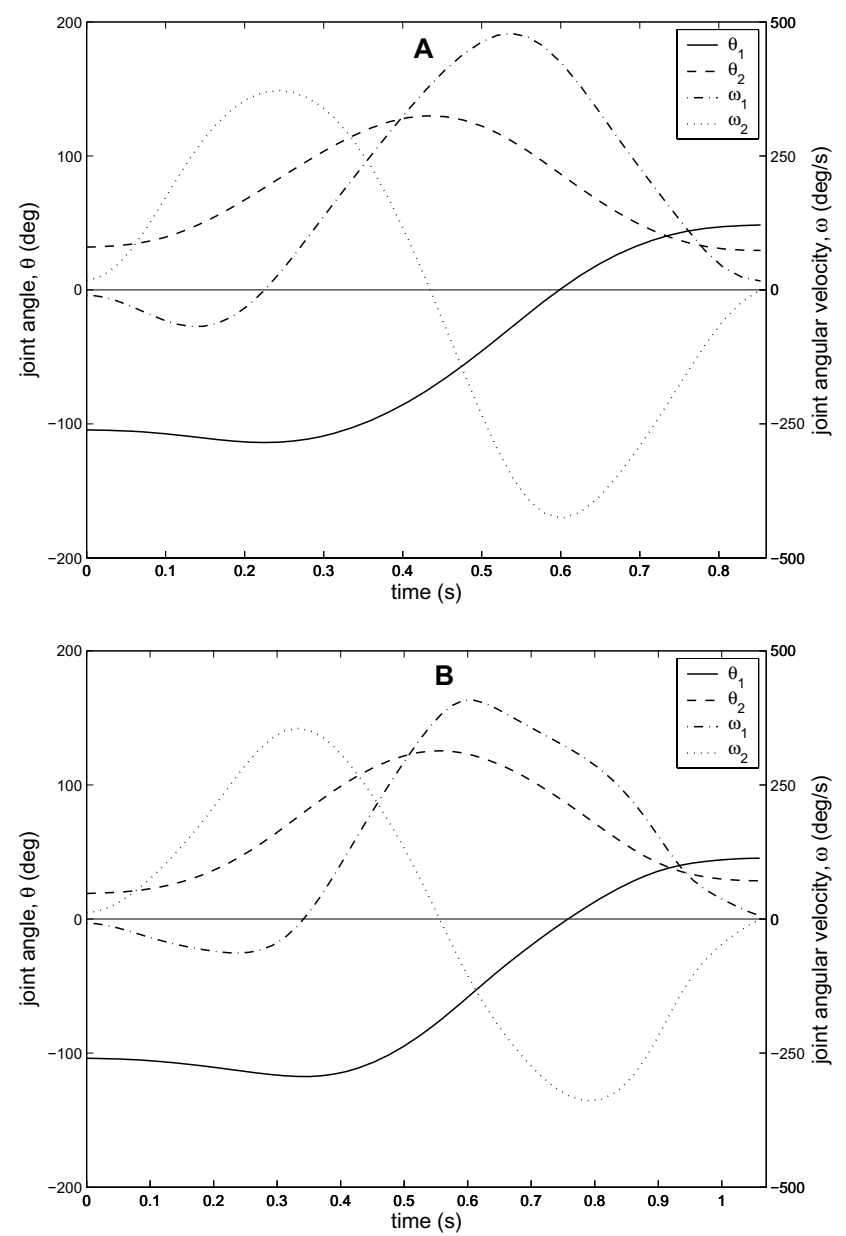

Figure 3 Joint angular displacements and joint angular velocities recorded for unloaded $(A)$ and loaded $(B)$ vertical arm movements. The solid and dashed lines represent shoulder $\left(\theta_{1}\right)$ and elbow $\left(\theta_{2}\right)$ joint angles, respectively. The dashdotted and dotted lines are the joint angular velocities $\left(\omega_{i}=\dot{\theta}_{i} ; i=1,2\right)$ of the shoulder and elbow, respectively.

torques, T. Thus, by virtually 'turning off' the applied torques, one at a time, and subtracting the resultant acceleration from the acceleration of the hand (when both torques were included), the contribution of each joint torque was computed. Analogously, the contributions of gravity (G) and the velocity-interaction torques $(\mathbf{V})$ to the vertical acceleration of the hand were computed by subtracting the acceleration without each contributor, respectively, from the total acceleration of the hand. Equations (5) to (7) were also generated in symbolic form using DynaFlex, with additional substitutions and simplifications performed using the Maple computer algebra system.

\section{Results}

Unrestrained vertical arm movements produced curved trajectories of the hand, whether or not the hand was loaded with an additional mass (compare trajectories of the hand in Figure 2A and 2B). These results are consistent with those
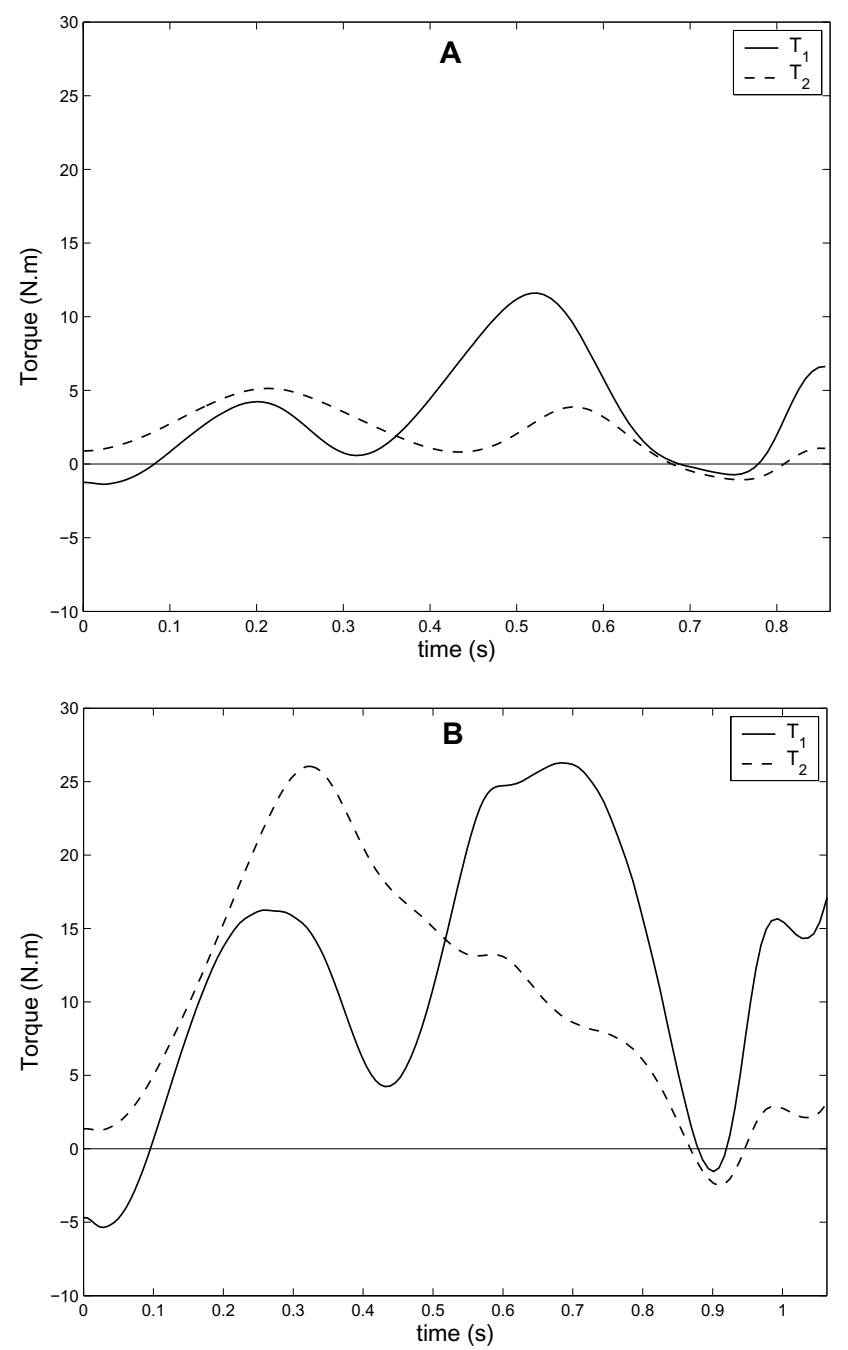

Figure 4 Net muscle torques calculated for the shoulder and elbow joints during unloaded (A) and loaded (B) vertical arm movements. The solid lines correspond to the net shoulder torques $\left(T_{1}\right)$, and the dashed lines to the net elbow torques $\left(T_{2}\right)$. Joint torques were computed using the measured joint kinematics and by applying the inverse-dynamics approach (see the section Methods).

of Atkeson and Hollerbach (1985), who found that movements in the horizontal plane resulted in substantially straight paths, but movements between set targets in the vertical plane were more curved.

The shoulder experienced mainly flexion, while the elbow underwent both flexion and extension (Figure 3). Movements of the joints were finely timed, beginning with flexion of the elbow and followed by simultaneous flexion and extension of the shoulder and elbow, respectively. The patterns of joint angular displacements and velocities were invariant to changes in load. The magnitudes and shapes of the shoulder joint angles were very similar for the unloaded and loaded arm movements, with elbow joint angles being virtually identical (compare solid and dashed curves in Figure 3A). There was close similarity in the shapes of the 

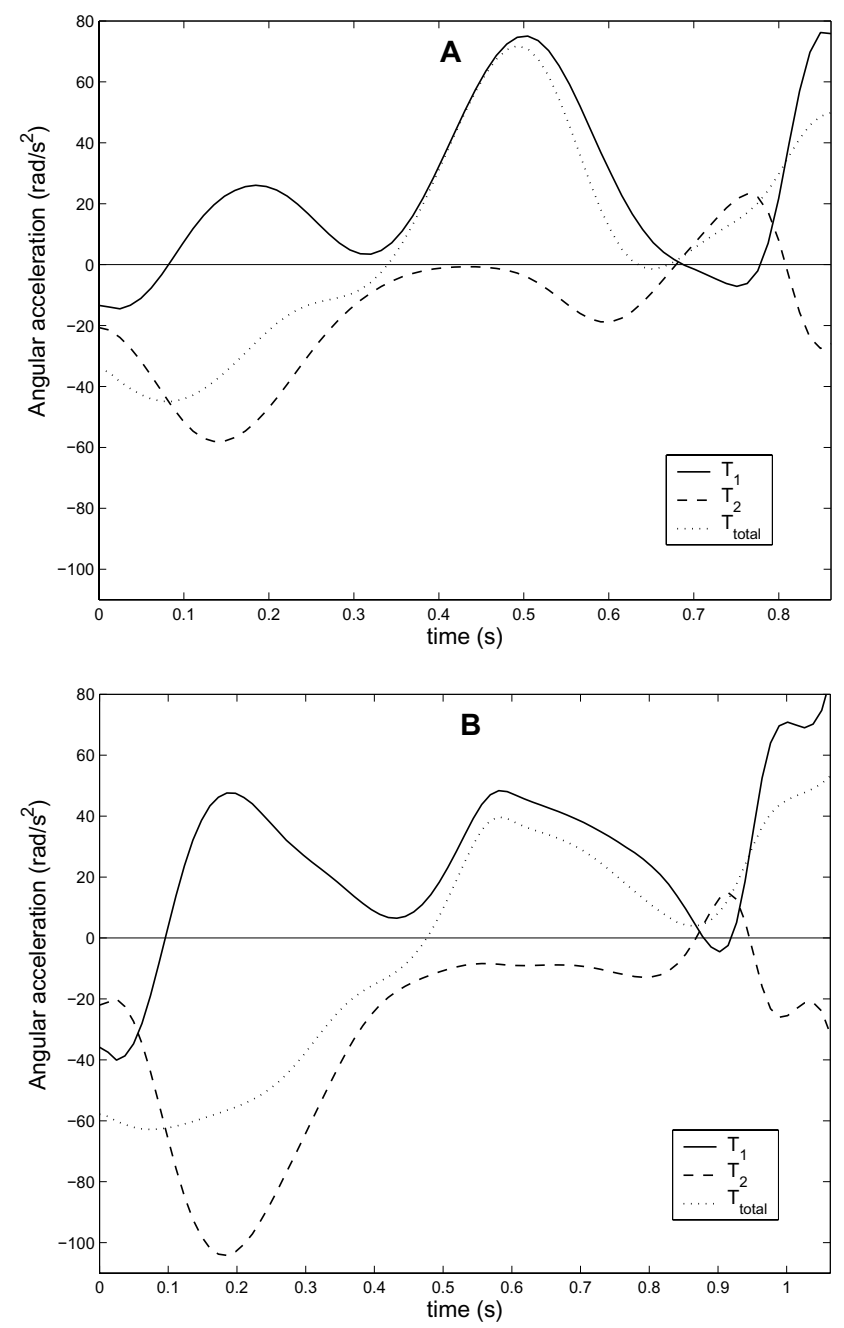

Figure 5 Contributions of the net muscle torques acting at the shoulder and elbow to the angular acceleration of the shoulder joint during unloaded $(A)$ and loaded (B) vertical arm movements. The solid lines represent the joint angular acceleration of the shoulder induced by the net muscle torque at the shoulder $\left(T_{1}\right)$; the dashed lines represent the joint angular acceleration of the shoulder induced by the net muscle torque at the elbow $\left(T_{2}\right)$; and the dotted lines represent the joint angular acceleration of the shoulder induced by the net muscle torques at the shoulder and the elbow combined $\left(T_{\text {total }}\right)$.

corresponding joint velocities as well (compare dotted and dot-dashed curves in Figure 3B). Again, these results are consistent with those reported by Atkeson and Hollerbach (1985), who found that the tangential velocity profile in vertical arm movement, when normalised by time and peak velocity, was invariant to changes in external load. The only notable effect of load on the kinematics of arm movement found in our experiments was an increase in movement time of approximately $25 \%$ (compare time scales in Figures $3 \mathrm{~A}$ and $3 \mathrm{~B})$.

While the pattern of joint kinematics was invariant to a hand-held load, the dynamics of arm movement were not. In the unloaded state, a three-phase pattern of joint torque was evident at both the shoulder and elbow, with peak shoulder torque being more than twice that of the peak elbow
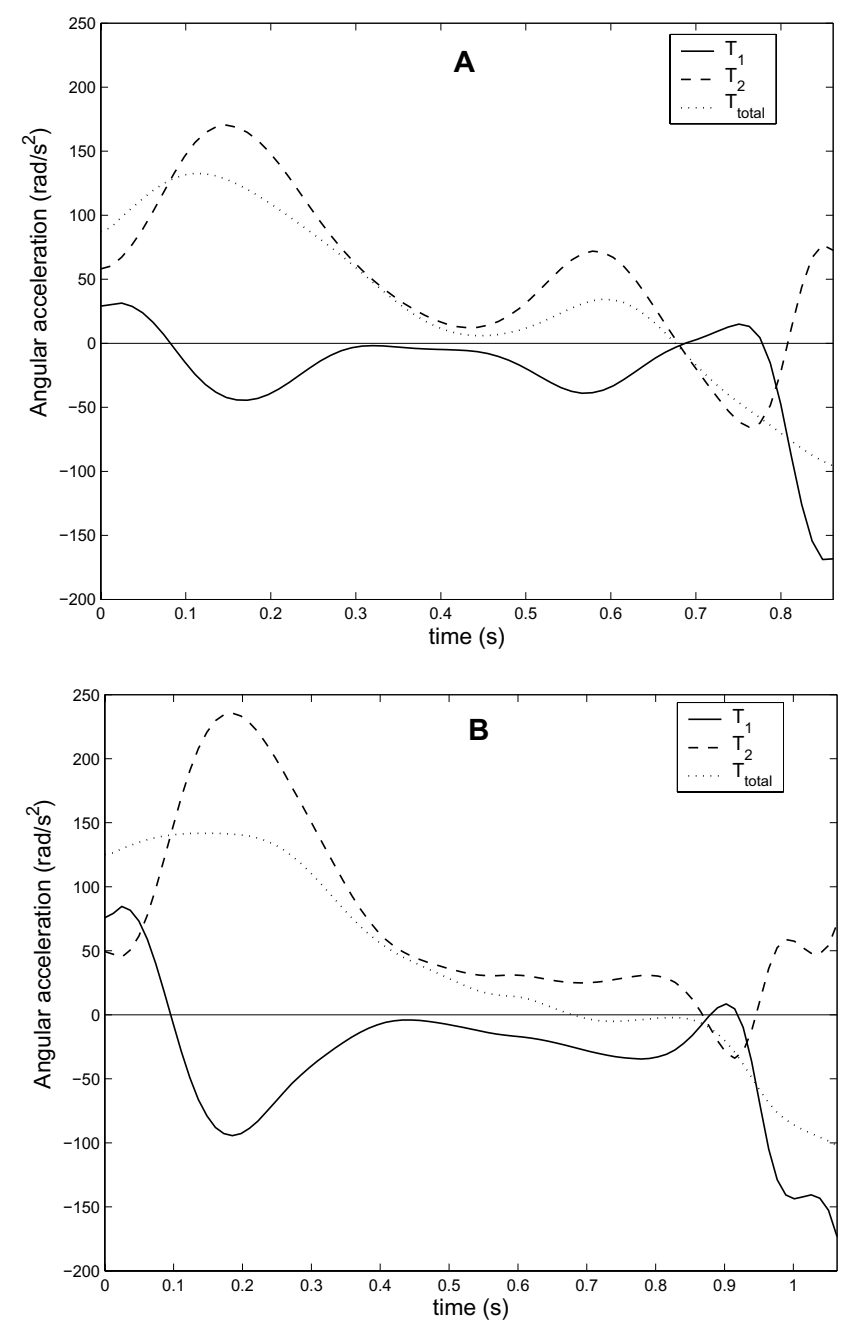

Figure 6 Contributions of the net muscle torques acting at the shoulder and elbow to the angular acceleration of the elbow joint during unloaded $(A)$ and loaded (B) vertical arm movements. The solid lines represent the joint angular acceleration of the elbow induced by the net muscle torque at the shoulder $\left(T_{1}\right)$; the dashed lines represent the joint angular acceleration of the elbow induced by the net muscle torque at the elbow $\left(T_{2}\right)$; and the dotted lines represent the joint angular acceleration of the elbow induced by the net muscle torques at the shoulder and the elbow combined $\left(T_{\text {total }}\right)$.

torque (compare solid and dashed lines in Figure 4A). In contrast, peak shoulder and elbow torques were nearly equal when a $5-\mathrm{kg}$ load was carried in the hand (compare solid and dashed lines in Figure 4B). The profile of shoulder torque remained tri-phasic during the loaded movement, while that of the elbow torque was more bimodal, with the second peak being obscured by the breadth of the larger initial peak (compare dashed lines in Figure 4B). Note that the subsequent increase in shoulder and elbow torque at the end of the movements arose from the constraint that the mass was to be brought to rest at the final elevation.

A net muscle torque developed at one joint accelerated both joints in the model simultaneously. Furthermore, each net muscle torque induced predominantly opposite actions at the shoulder and elbow (compare solid and dashed lines 

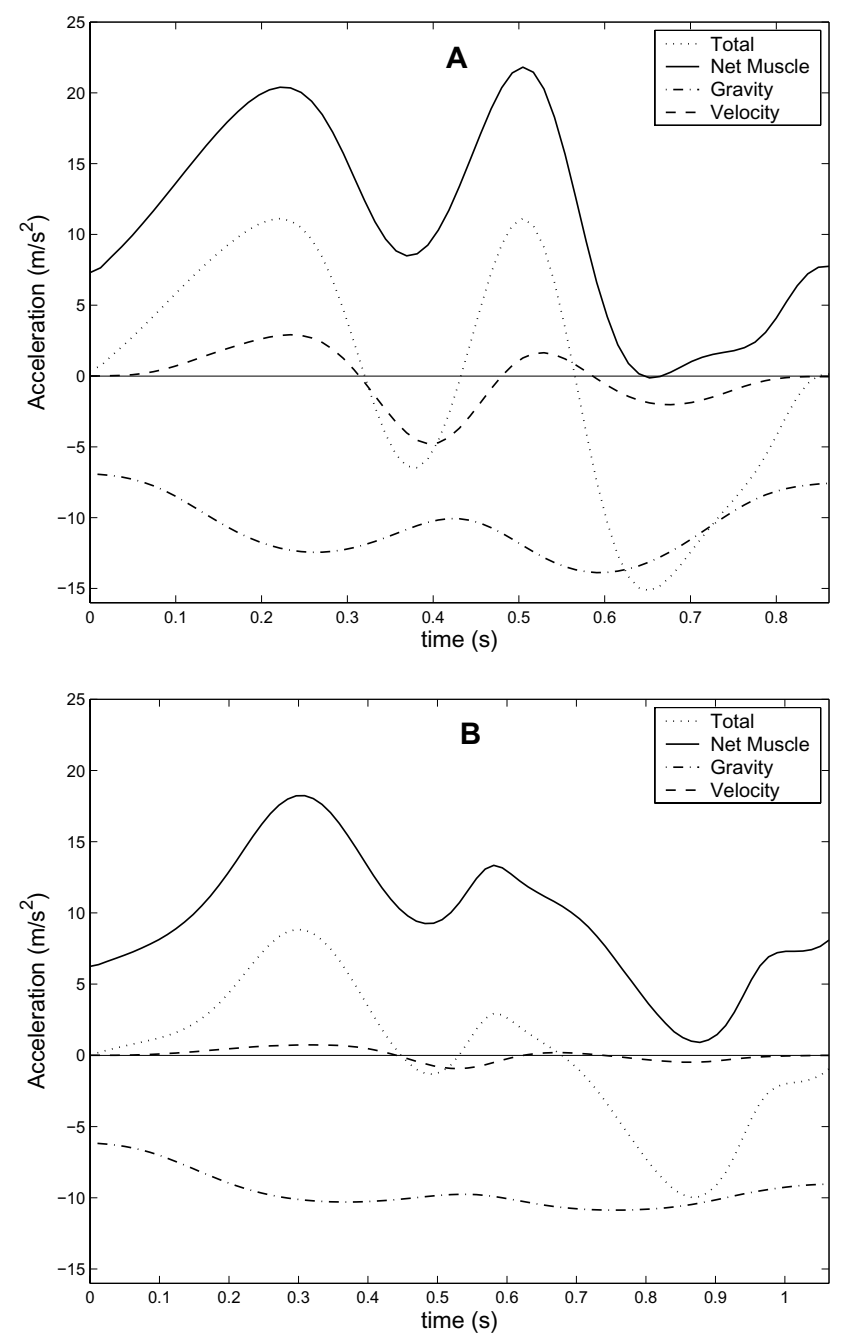

Figure 7 Contributions of the net muscle torques, the gravitational torques and the velocity-interaction torques to the total vertical acceleration of the hand during unloaded (A) and loaded (B) vertical arm movements. The solid lines represent the vertical acceleration of the hand induced by the net muscle torques at the shoulder and elbow combined; the dashed-dot lines represent the vertical acceleration of the hand induced by the gravitational torques; and the dashed lines represent the vertical acceleration of the hand induced by the velocity-interaction torques. Dotted line indicates the total of all contributions.

in Figures 5A and 5B, and in Figures 6A and 6B). For example, at most times during the movement, a net torque acting at the shoulder accelerated the shoulder into flexion and the elbow into extension (compare solid and dashed lines in Figures 5A and 5B). At the beginning of the movement, however, the reverse was true, with the shoulder torque, which was extensor (Figure 4 , shoulder at time $\mathrm{t}=0$ ) inducing the elbow into flexion (solid lines in Figure 6).

Muscle action dominated the vertical acceleration of the hand (Figure 7). Gravitational torques accelerated the hand downward, as one would expect, while the muscle torques provided a balancing upward acceleration (compare solid and dot-dashed curves in Figures 7A and 7B). Past the twothirds mark in the task, the contributions of the applied muscle torques faded (Figure 4) so much that gravity became
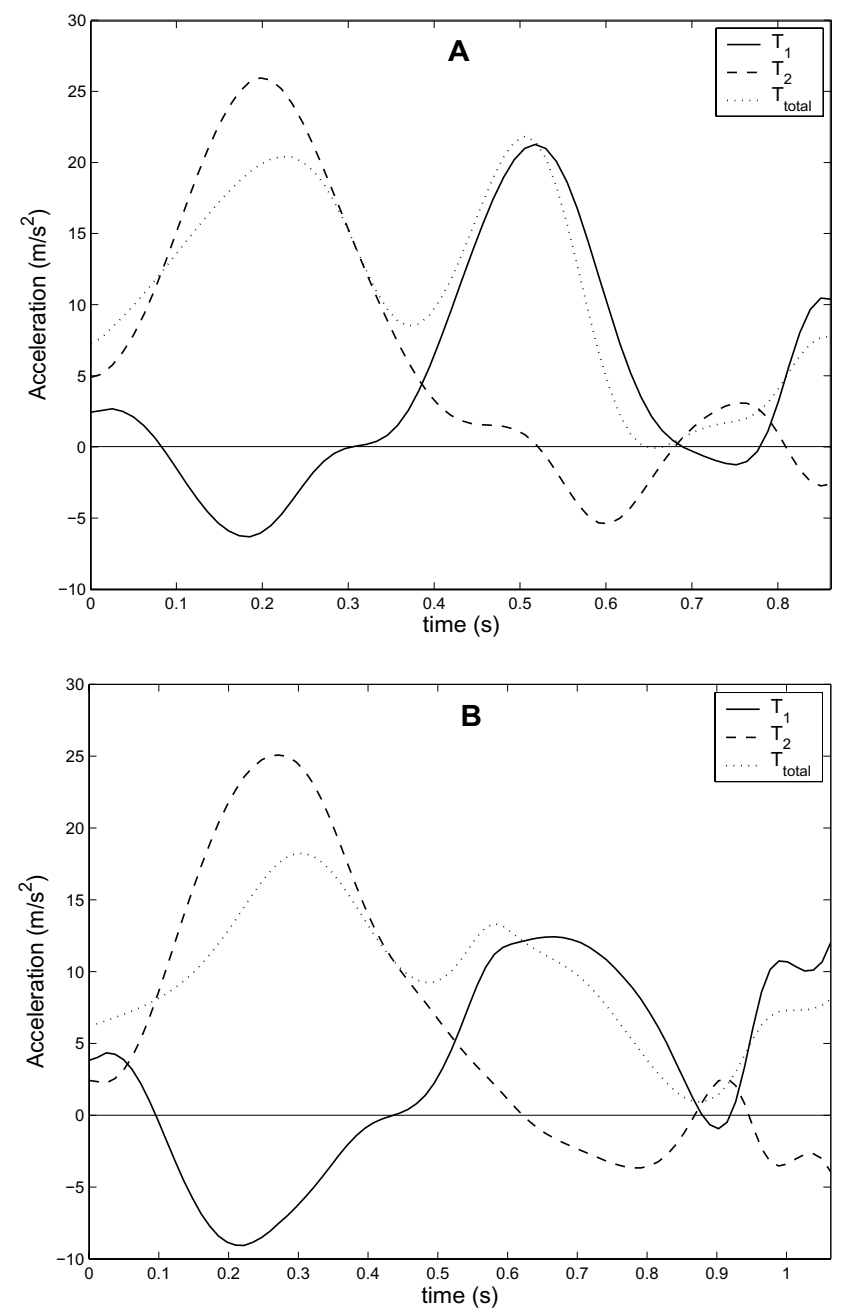

Figure 8 Contributions of the net muscle torques acting at the shoulder and elbow to the vertical acceleration of the hand during unloaded (A) and loaded (B) vertical arm movements. The solid lines represent the vertical acceleration of the hand induced by the net muscle torque at the shoulder $\left(T_{1}\right)$; the dashed lines represent the vertical acceleration of the hand induced by the net muscle torque at the elbow $\left(T_{2}\right)$; and the dotted lines represent the vertical acceleration of the hand induced by the net muscle torques at the shoulder and the elbow combined $\left(T_{\text {total }}\right)$.

the primary decelerator of the hand at the end of the movement. Thus, the muscle torques increased rapidly at the end of the movement to counterbalance the effect of gravity. An intriguing result is that the profile of the gravity contribution emulates that of the hand trajectory (compare gravity in Figure 7 with the hand paths shown in Figure 2), and that the magnitude of the gravitational contribution to the hand acceleration was approximately $15 \%$ less when the hand was loaded (compare dash-dot lines in Figures 7A and $7 \mathrm{~B}$ ). This suggests that part of the strategy used to coordinate arm movement in the presence of a load was to reduce the gravitational torques at the joints.

The velocity interaction torques remained relatively small throughout the course of the movement (dashed lines in Figure 7). In fact, the contribution of the velocity 
interaction torques to vertical acceleration of the hand decreased when the hand was loaded with a $5-\mathrm{kg}$ mass (compare dotted lines in Figures 7A and 7B).

The patterns of torque-induced hand accelerations were similar for the unloaded and loaded movements. Both the shoulder and elbow resisted gravity by accelerating the hand upward; however, both joints also contributed to downward accelerations of the hand for brief periods to oppose the relatively large upward acceleration induced at the neighbouring joint (compare solid and dashed lines in Figures $8 \mathrm{~A}$ and $8 \mathrm{~B}$ ). The hand was always accelerated upward by the elbow torque first; the net torque at the shoulder then acted to provide the upward acceleration following the decline of the elbow torque. Peak upward acceleration of the hand induced by the elbow was always larger than that induced by the shoulder (compare solid and dashed lines in Figures 8A and 8B).

The same control sequence was employed in the loaded movement, except that five times as much elbow flexor torque was required to produce the same amount of hand acceleration initially (compare dashed lines in Figures 4 and 8 , respectively). In contrast, shoulder flexor torque increased by only twice as much in the loaded movement and was almost equal to the peak torque developed by the elbow. The shoulder torque contribution to the hand acceleration was $50 \%$ less in the loaded movement but was also slower to decrease (solid line in Figure 8B).

\section{Discussion}

The purpose of this study was threefold: first, to evaluate the relative contributions of the net muscle torques, gravitational torques and velocity interaction torques in an unrestrained vertical movement of the arm; second, to understand how movements of the shoulder and elbow are coordinated during elevation of the arm in the sagittal plane; and third, to study the effect of external load on multi-joint coordination in the upper limb.

Atkeson and Hollerbach (1985) measured the kinematics of unrestrained vertical arm movement between point targets under varying conditions of speed and hand-held load. Soechting and Flanders (1997), Flanders et al $(1994,1996)$ and Gottlieb et al (1996) reported on the dynamics (ie patterns of torque production and muscle activation) of various pointing tasks, including upwardly directed movements in the vertical plane. The task analysed in the present study was most similar to that described by Atkeson and Hollerbach (1985). Our subject also performed upwardly directed movements with no strict constraints placed on the initial or final positions of the hand, the intermediate hand path and the movement speed.

Before interpreting the results, it is important to discuss some of the limitations of this study. First, there are limitations in the experimental methods employed, most specifically in the ability to estimate the derivatives of the position data. The choice was made to mitigate the lack of direct measures of segmental velocities and accelerations by determining a 'best' estimate of the joint accelerations using a feedback controller. Although this provides torques and accelerations that are consistent with the position data, there is a small likelihood that these accelerations do not precisely reflect the subject's performance. Second, a relatively simple two-segment, two-degree of freedom model was used to represent the dynamics of arm movement. This type of model has been studied extensively by previous investigators, eg Hollerbach and Flash (1982) and Gottlieb et al (1996), and therefore provides a significant basis for comparison with previous results. The human upper limb, with more joints and degrees of freedom than are incorporated into our model, has the potential to exhibit more dynamic coupling effects, such that dynamical interactions between the joints could be quite different. A vertical reaching movement that was limited to the sagittal plane was used to reduce the influence of out-of-plane accelerations that could not be captured in this model. Finally, the experimental results were obtained from a single healthy test subject, and therefore the interpretation of these results cannot be generalised to the population at large without further studies.

Hollerbach and Flash (1982) evaluated the significance of the inertial- and velocity-interaction torques as subjects performed a constrained movement of the shoulder and elbow in the horizontal plane (ie with the effect of gravity removed). Although the sizes of these interaction torques were not explicitly computed in the present study, it can be predicted that the inertial-interaction torques, which are proportional to the joint angular accelerations, are as significant for upwardly directed arm movements as for movements in the horizontal plane (Hollerbach and Flash 1982; Gribble and Ostry 1999). Our results indicate that the velocity terms contribute relatively little to the total vertical acceleration of the hand, and that these contributions actually decrease when an external load is added to the hand (compare total and velocity in Figures 7A and 7B). This suggests, in turn, that the vertical acceleration of the hand is most affected by the angular accelerations of the joints. 
Gravity and muscle action dominated the vertical acceleration of the hand in the loaded and unloaded arm movements, and the contribution of the velocity interaction torques remained relatively small throughout (compare net muscle and velocity in Figure 7). These findings are very consistent with previous results obtained for the lower limb in jumping (Pandy and Zajac 1991), cycling (Fregly and Zajac 1996) and normal walking (Anderson and Pandy 2003; Pandy 2003).

Using a four-segment planar model of the body, Pandy and Zajac (1991) calculated the contributions of leg-muscle forces, gravitational forces, and velocity-interaction forces (ie Coriolis and centripetal forces) to the total instantaneous power developed during a maximum-height jump. They found that the leg muscles contributed nearly all of the power delivered to the body for nearly the entire ground contact phase of the jump. Fregly and Zajac (1996) found that the leg muscles supplied practically all of the power to drive a bicycle crank against a frictional load, while the contribution of the velocity-interaction forces remained relatively small. Very recently, Anderson and Pandy (2003) used a much more detailed three-dimensional, muscle-actuated model of the body to compute muscle, gravitational and velocity contributions to the vertical acceleration of the centre of mass of the body during normal walking (see also Pandy 2003). They found that the leg muscles induced a relatively large upward acceleration on the centre of mass to counteract the nearly constant downward acceleration induced by gravity (see Figure 6 in Anderson and Pandy 2003). Results obtained in the present study for arm movement in the vertical plane are analogous.

The muscles of the shoulder and elbow acted synergistically to elevate the arm in the sagittal plane. The elbow flexors were the prime movers of the arm in the first phase of the movement, while the shoulder flexors dominated the second phase (compare solid and dashed lines in Figures 4 and 8). Calculations of the joint angular accelerations induced by the net muscle torques revealed a number of interesting features of the coordination strategy adopted in this task. Perhaps most significantly, a net muscle torque applied at one joint usually had opposite effects at the shoulder and elbow (compare solid and dashed lines in Figures 5, 6 and 8). For example, net flexor torques were applied at the shoulder and elbow for practically the entire movement (solid and dashed lines in Figure 4). The effect of a flexor torque at the shoulder was to accelerate the shoulder into flexion but the elbow into extension (solid lines in Figures 5 and 6, respectively). Similarly, the effect of a flexor torque at the elbow was to accelerate the elbow into flexion but the shoulder into extension (dashed lines in Figures 5 and 6). Interestingly, the pattern of elbow flexion followed by shoulder flexion and then elbow extension has also been observed in minimum-time manoeuvres of a planar robot arm movement (Geering et al 1996; Heilig and McPhee 1999).

It was also found that, at times, the torque applied at a primary joint had a more powerful effect on the acceleration of a secondary joint than the torque applied at the secondary joint itself. In the first phase of the movement, for example, the net flexor torque applied at the shoulder accelerated the shoulder into flexion (solid lines in Figure 5). However, the net flexor torque applied by the elbow (prime mover) simultaneously accelerated the shoulder into extension with more vigour (dashed lines in Figure 5), and so the net action at the shoulder was an acceleration into extension (dotted lines in Figure 5). This result is perhaps easily understood here because the inertial interactions between two segments are relatively intuitive. When a model has more than two segments, however, an analysis like the one undertaken in this study can often lead to conclusions about multi-joint coordination that are not only much more complex but often counter-intuitive as well (Zajac and Gordon 1989; Pandy 2001).

Finally, it was found that multi-joint coordination in a vertically directed arm movement is quite invariant to changes in external load in terms of both the kinematics and applied torque sequencing. The path of the hand and the corresponding joint angular displacements of the shoulder and elbow were nearly the same in the unloaded and loaded movements (Figures 2 and 3 ). In addition, the pattern of vertical acceleration induced at the hand was very similar with and without the hand-held load (Figure 8). The muscles of the elbow accelerated the hand upward at the beginning of arm movement, whereas the muscles of the shoulder became the prime movers only after the elbow had reached maximum flexion. The fact that the hand trajectory was relatively unchanged when a load was added indicates that the motor system was able to devise a control strategy that effectively compensated for the dynamic effects introduced by the load. In fact, the small differences in kinematics can be directly attributed to the increase in inertia of the system and the necessity to increase the torque output accordingly. The increase in output, however, does not occur linearly, but in a compromise of more rapid and larger torque production (amplitude) with longer application periods (compare Figure 4B with 4A), resulting in a longer 
performance time. It was also evident that gravity both contributed less to hand acceleration and was more consistent (had less fluctuation) in the loaded case (Figure 7). Although there are relatively small differences in angular positions (Figure 3), they are significant enough to affect the gravitational torques at the joints, thereby reducing some of the demand on the applied torques in the loaded case (opposed to no variation in joint angles, where gravity would have had the identical effect). One could also speculate that the curved paths in unrestrained vertical movements when compared with linear paths in horizontal movements (Atkeson and Hollerbach 1985) are a consequence of path adaptations to reduce contributions from gravitational torques. Therefore, it would appear that the motor control system plans to replicate a set pattern for the arm movement, evident in the kinematics (Figures 2 and 3 ), but the differences in the dynamics forces the controller to make adaptations in the 'desired path' and to compensate applied muscle torques via signal gain and period adjustments.

\section{Acknowledgements}

Supported in part by the Engineering Research Centers Program of the National Science Foundation under Award nr EEC9876363 and the Natural Sciences and Engineering Research Council of Canada. We thank Dr Richard Wells at the University of Waterloo for his assistance with data collection and the use of laboratory facilities.

\section{Appendix 1}

The symbols identifying the parameters of the planar arm model (Figure 1A) are identified and their values defined herein. The upper arm, forearm and hand masses are $m_{1}, m_{2}$ and $m_{3}$, and their corresponding inertias are $I_{1}, I_{2}$ and $I_{3}$. The length of the upper arm and forearm are $l_{1}$ and $l_{2}$, and the corresponding shoulder and elbow torques are $T_{1}$ and $T_{2}$. In the linear-graph representation (Figure 1B), all edges are uniquely numbered. Rigid-arm elements describing the fixed distances between segment centres of mass and joints are labelled $r_{4-7}$, corresponding to the shoulder joint to upper-arm centre of mass, upper-arm centre of mass to the elbow joint, elbow joint to forearm centre of mass and forearm centre of mass to wrist joint, respectively. Joints are represented by $\mathrm{h}_{8-9}$ and $\mathrm{w}_{10}$, which are two hinge joints at the shoulder and elbow, and a weld joint of the hand $\left(m_{3}\right)$ to the wrist. The applied joint torques ( $T_{1}$ and $T_{2}$ in Figure $\left.1 \mathrm{~A}\right)$ appear as $T_{11}$ and $T_{12}$ in the corresponding graph. Mass values were obtained from Kingma et al (1996), and lengths from the average lengths of the reconstructed segment kinematics, while moments of inertia were calculated using a cylindrical approximation. Please note that $I_{1}$ is the inertia of the upper limb about the shoulder joint, whereas the inertias $I_{2}$ and $I_{3}$ are about their centre of mass. Parameter values are as follows:

$$
\begin{aligned}
& m_{1}=2.4 \mathrm{~kg} \quad m_{2}=1.1 \mathrm{~kg} \quad m_{3}=0.45 \mathrm{~kg} \\
& I_{1}=0.0636 \mathrm{~kg} \mathrm{~m}^{2} \quad I_{2}=0.0074 \mathrm{~kg} \mathrm{~m}^{2} \quad I_{3}=0.0010 \mathrm{~kg} \mathrm{~m}^{2} \\
& l_{1}=r_{4}+r_{5}=0.3257 \mathrm{~m} \quad l_{2}=r_{6}+r_{7}=0.2845 \mathrm{~m} \\
& r_{4}=0.1257 \mathrm{~m} \quad r_{5}=0.2000 \mathrm{~m} \quad r_{6}=0.1500 \mathrm{~m} \quad r_{7}=0.1345 \mathrm{~m}
\end{aligned}
$$

Note, in the loaded case, the $5-\mathrm{kg}$ mass was added as a point mass at the hand, such that $m_{3}{ }^{\prime}=5.45 \mathrm{~kg}$.

The dynamical equations of motion for the two-segment planar model of the arm shown in Figure 1 are given below. These equations were derived using a graph-theoretic approach identified in the text (see Shi and McPhee (2000) and Seth (2000) for details). The general form of the dynamical equations of motion can be written as:

$$
\mathbf{M}(\boldsymbol{\theta}) \ddot{\boldsymbol{\theta}}=\mathbf{V}(\boldsymbol{\theta}, \dot{\theta})-\mathbf{G}(\boldsymbol{\theta})+\mathbf{T}
$$

where:

$$
\begin{gathered}
\boldsymbol{\theta}=\left[\theta_{1}, \theta_{2}\right]^{\mathrm{T}} \\
\mathbf{M}(\boldsymbol{\theta})=\left[\begin{array}{cc}
n_{1}+n_{2} \cos \left(\theta_{2}\right) & n_{3}+\frac{1}{2} n_{2} \cos \left(\theta_{2}\right) \\
n_{3}+\frac{1}{2} n_{2} \cos \left(\theta_{2}\right) & n_{3}
\end{array}\right] \\
n_{1}=\left(m_{2}+m_{3}\right) l_{1}^{2}+m_{2} r_{6}^{2}+m_{3} l_{2}^{2}+I_{1}+I_{2}+I_{3} \\
n_{2}=2\left(m_{2} r_{6}+m_{3} l_{2}\right) l_{1} \\
n_{3}=m_{2} r_{6}^{2}+m_{3} l_{2}^{2}+I_{2}+I_{3} \\
\mathbf{V}(\boldsymbol{\theta}, \dot{\boldsymbol{\theta}})=\left\{\begin{array}{c}
\frac{1}{2} n_{2} \sin \left(\theta_{2}\right)\left(\left(\dot{\theta}_{2}\right)^{2}+2 \dot{\theta}_{1} \dot{\theta}_{2}\right) \\
-\frac{1}{2} n_{2} \sin \left(\theta_{2}\right)\left(\dot{\theta}_{1}\right)^{2}
\end{array}\right\}
\end{gathered}
$$




$$
\mathbf{G}(\boldsymbol{\theta})=g\left\{\begin{array}{c}
\left(m_{1} r_{4}+\left(m_{2}+m_{3}\right) l_{1}\right) \cos \left(\theta_{1}\right)+\left(m_{2} r_{6}+m_{3} l_{2}\right) \cos \left(\theta_{1}+\theta_{2}\right) \\
\left(m_{2} r_{6}+m_{3} l_{2}\right) \cos \left(\theta_{1}+\theta_{2}\right)
\end{array}\right\}
$$

where $g=9.81 \mathrm{~m} / \mathrm{s}^{2}$

Equation (A1) can be solved for the joint angular accelerations as follows:

$$
\ddot{\theta}=\mathbf{M}^{-1}(\mathbf{V}(\boldsymbol{\theta}, \dot{\theta})-\mathbf{G}(\boldsymbol{\theta})+\mathbf{T})
$$

where the inverse of the mass matrix, $\mathbf{M}^{-1}$, can be written as:

$$
\begin{aligned}
& \mathbf{M}^{-1}=\frac{\left[\begin{array}{cc}
n_{3} & -\left(n_{3}+\frac{1}{2} n_{2} \cos \left(\theta_{2}\right)\right) \\
-\left(n_{3}+\frac{1}{2} n_{2} \cos \left(\theta_{2}\right)\right) & n_{1}+n_{2} \cos \left(\theta_{2}\right)
\end{array}\right]}{|\mathbf{M}(\theta)|} \\
& |\mathbf{M}(\theta)|=n_{1} n_{3}-n_{3}^{2}+\left(\frac{1}{2} n_{2} \cos \left(\theta_{2}\right)\right)^{2}
\end{aligned}
$$

Equation (A2) was used to compute the contributions of the net muscle torques to the joint angular accelerations of the shoulder and elbow (see Figures 5 and 6). Rewriting equation (A1) to isolate the net muscle (joint) torques, we have:

$$
\begin{aligned}
\mathbf{T}= & \mathbf{M}(\boldsymbol{\theta}) \ddot{\boldsymbol{\theta}}-(\mathbf{V}(\boldsymbol{\theta}, \dot{\boldsymbol{\theta}})-\mathbf{G}(\boldsymbol{\theta})) \\
T_{1}= & \left(n_{1}+n_{2} \cos \left(\theta_{2}\right)\right) \ddot{\theta}_{1}+\left(n_{3}+\frac{1}{2} n_{2} \cos \left(\theta_{2}\right)\right) \ddot{\theta}_{2}-\frac{1}{2} n_{2} \sin \left(\theta_{2}\right) \dot{\theta}_{2}^{2}-n_{2} \sin \left(\theta_{2}\right) \dot{\theta}_{1} \dot{\theta}_{2}+\left(\left(m_{1} r_{4}+\left(m_{2}+m_{3}\right) l_{1}\right) \cos \left(\theta_{1}\right)\right. \\
& \left.+\left(m_{2} r_{6}+m_{3} l_{2}\right) \cos \left(\theta_{1}+\theta_{2}\right)\right) g \\
T_{2}= & \left(n_{3}+\frac{1}{2} n_{2} \cos \left(\theta_{2}\right)\right) \ddot{\theta}_{1}+n_{3} \ddot{\theta}_{2}+\frac{1}{2} n_{2} \sin \left(\theta_{2}\right) \dot{\theta}_{1}^{2}+g\left(m_{2} r_{6}+m_{3} l_{2}\right) \cos \left(\theta_{1}+\theta_{2}\right)
\end{aligned}
$$

Equations (A3a) and (A3b) are the explicit inverse dynamics equations for the torques in the planar two-segment model of the arm. These equations were used to compute the net muscle torques at the shoulder and elbow shown in Figure 4. Next, we derive the relationships between the net muscle torques and the vertical acceleration of the hand. The absolute position of the hand, in ground coordinates, can be written as:

$$
\mathbf{p}=\mathbf{R}\left(\theta_{1}\right)\left\{\begin{array}{l}
l_{1} \\
0
\end{array}\right\}+\mathbf{R}\left(\theta_{1}+\theta_{2}\right)\left\{\begin{array}{l}
l_{2} \\
0
\end{array}\right\}
$$

where the matrices $\mathbf{R}\left(\theta_{1}\right)=\left[\begin{array}{rr}\cos \left(\theta_{1}\right) & -\sin \left(\theta_{1}\right) \\ \sin \left(\theta_{1}\right) & \cos \left(\theta_{1}\right)\end{array}\right]$ and $\mathbf{R}\left(\theta_{1}+\theta_{2}\right)=\left[\begin{array}{rr}\cos \left(\theta_{1}+\theta_{2}\right) & -\sin \left(\theta_{1}+\theta_{2}\right) \\ \sin \left(\theta_{1}+\theta_{2}\right) & \cos \left(\theta_{1}+\theta_{2}\right)\end{array}\right]$ are the rotation matrices of the upper arm and forearm, respectively, relative to the ground. Differentiating equation (A4) with respect to time yields the absolute velocity of the hand:

$$
\dot{\mathbf{p}}=\mathbf{R}^{\prime}\left(\theta_{1}\right)\left\{\begin{array}{l}
l_{1} \\
0
\end{array}\right\} \dot{\theta}_{1}+\mathbf{R}^{\prime}\left(\theta_{1}+\theta_{2}\right)\left\{\begin{array}{l}
l_{2} \\
0
\end{array}\right\}\left(\dot{\theta}_{1}+\dot{\theta}_{2}\right)
$$

where the matrices $\mathbf{R}^{\prime}\left(\theta_{1}\right)=\left[\begin{array}{cc}-\sin \left(\theta_{1}\right) & -\cos \left(\theta_{1}\right) \\ \cos \left(\theta_{1}\right) & -\sin \left(\theta_{1}\right)\end{array}\right]$ and $\mathbf{R}^{\prime}\left(\theta_{1}+\theta_{2}\right)=\left[\begin{array}{cc}-\sin \left(\theta_{1}+\theta_{2}\right) & -\cos \left(\theta_{1}+\theta_{2}\right) \\ \cos \left(\theta_{1}+\theta_{2}\right) & -\sin \left(\theta_{1}+\theta_{2}\right)\end{array}\right]$ are coefficient matrices defined by $\mathbf{R}^{\prime}=\frac{\partial \mathbf{R}}{\partial \theta}$.

Differentiating the hand velocity equation (A5), and using $\mathbf{R}^{\prime \prime}=-\mathbf{R}$, gives an expression for the acceleration of the hand, thus:

$$
\ddot{\mathbf{p}}=\mathbf{R}^{\prime}\left(\theta_{1}\right)\left\{\begin{array}{l}
l_{1} \\
0
\end{array}\right\} \ddot{\theta}_{1}-\mathbf{R}\left(\theta_{1}\right)\left\{\begin{array}{l}
l_{1} \\
0
\end{array}\right\}\left(\dot{\theta}_{1}\right)^{2}+\mathbf{R}^{\prime}\left(\theta_{1}+\theta_{2}\right)\left\{\begin{array}{l}
l_{2} \\
0
\end{array}\right\}\left(\ddot{\theta}_{1}+\ddot{\theta}_{2}\right)-\mathbf{R}\left(\theta_{1}+\theta_{2}\right)\left\{\begin{array}{c}
l_{2} \\
0
\end{array}\right\}\left(\dot{\theta}_{1}+\dot{\theta}_{2}\right)^{2}
$$

Finally, taking only the second component of the hand acceleration column matrix, $\ddot{\mathbf{p}}$, yields the vertical acceleration of the hand:

$$
\ddot{y}=l_{1} \cos \left(\theta_{1}\right) \ddot{\theta}_{1}-l_{1} \sin \left(\theta_{1}\right)\left(\dot{\theta}_{1}\right)^{2}+l_{2} \cos \left(\theta_{1}+\theta_{2}\right)\left(\ddot{\theta}_{1}+\ddot{\theta}_{2}\right)-l_{2} \sin \left(\theta_{1}+\theta_{2}\right)\left(\dot{\theta}_{1}+\dot{\theta}_{2}\right)^{2}
$$

and collecting $\ddot{\boldsymbol{\theta}}$ and $\dot{\boldsymbol{\theta}}^{2}$ terms, yields:

$$
\ddot{y}=\left[\left(l_{1} \cos \left(\theta_{1}\right)+l_{2} \cos \left(\theta_{1}+\theta_{2}\right)\right) \quad l_{2} \cos \left(\theta_{1}+\theta_{2}\right)\right] \ddot{\boldsymbol{\theta}}-\left[\left(l_{1} \sin \left(\theta_{1}\right)+l_{2} \sin \left(\theta_{1}+\theta_{2}\right)\right) \quad l_{2} \sin \left(\theta_{1}+\theta_{2}\right)\right] \dot{\boldsymbol{\theta}}^{2}-2 l_{2} \sin \left(\theta_{1}+\theta_{2}\right) \dot{\theta}_{1} \dot{\theta}_{2}
$$

Substituting the expressions for the angular accelerations from equation (A2) gives the vertical acceleration of the hand as a function only of the state of the system $(\boldsymbol{\theta}, \dot{\boldsymbol{\theta}})$ and applied net muscle torques, $\mathbf{T}$. 
Thus:

$$
\begin{aligned}
& \ddot{y}=\left[\left(l_{1} \cos \left(\theta_{1}\right)+l_{2} \cos \left(\theta_{1}+\theta_{2}\right)\right) \quad l_{2} \cos \left(\theta_{1}+\theta_{2}\right)\right]\left\{\mathbf{M}^{-1}(\mathbf{V}(\boldsymbol{\theta}, \dot{\boldsymbol{\theta}})-\mathbf{G}(\boldsymbol{\theta})+\mathbf{T})\right\} \\
& -\left[\left(l_{1} \sin \left(\theta_{1}\right)+l_{2} \sin \left(\theta_{1}+\theta_{2}\right)\right) \quad l_{2} \sin \left(\theta_{1}+\theta_{2}\right)\right] \dot{\theta}^{2}-2 l_{2} \sin \left(\theta_{1}+\theta_{2}\right) \dot{\theta}_{1} \dot{\theta}_{2}
\end{aligned}
$$

Equation (A8) was used to compute the contributions of the net muscle torques, the gravitational torques and the velocity-interaction torques to the vertical acceleration of the hand (see Figures 7 and 8).

\section{References}

Anderson FC, Pandy MG. 2003. Individual muscle contributions to support in normal walking. Gait Posture, 17:159-69.

Atkeson CG, Hollerbach JM. 1985. Kinematic features of unrestrained vertical arm movements. J Neurosci, 5:2318-30.

Biryukova EV, Roby-Brami A, Frolov AA et al. 2000. Kinematics of the human arm reconstructed from spatial tracking system recordings. J Biomech, 33:985-95.

Flanders M, Pellegrini JJ, Geisler SD. 1996. Basic features of phasic activation for reaching in vertical planes. Exp Brain Res, 110:67-79.

Flanders M, Pelligrini JJ, Soechting JF. 1994. Spatial/temporal characteristics of a motor pattern for reaching. $J$ Neurophysiol, 71: 811-13.

Flash T, Hogan N. 1985. The coordination of arm movements: an experimentally confirmed mathematical model. J Neurosci, 5: $1688-703$

Fregly BJ, Zajac FE. 1996. A state-space analysis of mechanical energy generation, absorption, and transfer during pedaling. J Biomech, 29:81-90.

Galloway JC, Koshland GF. 2002. General coordination of shoulder, elbow, and wrist dynamics during multijoint arm movements. Exp Brain Res, 142:163-80.

Garner BA, Pandy MG. 2001. Musculoskeletal model of the upper limb based on the visible human male dataset. Comput Methods Biomech Biomed Eng, 4:93-126.

Geering HP, Guzzella L, Hepner SA et al. 1996. Time-optimal motions of robots in assembly tasks. IEEE Trans Automatic Control, 31: 512-18.

Gottlieb GL, Song Q, Almeida GL et al. 1997. Directional control of planar human arm movement. J Neurophysiol, 78:2985-98.

Gottlieb GL, Song Q, Hong DA et al. 1996. Coordinating two degrees of freedom during human arm movement: load and speed invariance of relative joint torques. J Neurophysiol, 76:3196-206.

Gribble PL, Ostry DJ. 1999. Compensation for interaction torques during single- and multijoint limb movement. J Neurophysiol, 82:2310-26.

Heilig J, McPhee JJ. 1999. Determination of minimum-time maneuvers for a robotic manipulator using numerical optimization methods. Mech Struct Machines, 27:185-201.

Hollerbach J, Flash T. 1982. Dynamic interactions between limb segments during planar arm movement. Biol Cybern, 44:67-77.
Kingma I, Toussaint HM, Looze MP et al. 1996. Segment inertial parameter evaluation in two anthropometric models by application of dynamic linked segment model. J Biomech, 29:693-704.

Pandy MG. 2001. Computer modeling and simulation of human movement. Annu Rev Biomed Eng, 3:245-73.

Pandy MG. 2003. Simple and complex models for studying muscle function in walking. Proc R Soc Lond B Biol Sci, 358:1501-9.

Pandy MG, Zajac FE. 1991. Optimal muscular coordination strategies for jumping. J Biomech, 24:1-10.

Pandy MG, Zajac FE, Sim E et al. 1990. An optimal control model for maximum-height human jumping. J Biomech, 23:1185-98.

Risher DW, Shutte LM, Runge CF. 1997. The use of inverse dynamics solutions in direct dynamics simulations. J Biomech Eng, 119: 417-22.

Runge CF, Zajac FE III, Allum JH et al. 1995. Estimating net joint torques from kinesiological data using optimal linear system theory. IEEE Trans Biomed Eng, 42:1158-64.

Seth A. 2000. A predictive control method for human upper-limb motion: graph-theoretic modelling, dynamic optimization and experimental investigations [online]. Accessed 1 Oct 2003. URL: http:// etheses.uwaterloo.ca/display.cfm?ethesis_id=8

Shi P, McPhee JJ. 2000. Dynamics of flexible multibody systems using virtual work and linear graph theory. Multibody Syst Dyn, 4:335-81.

Shi P, McPhee JJ. 2002. Symbolic programming of a graph-theoretic approach to flexible multibody dynamics. Mech Struct Machines, 30:123-54.

Slotine JJ, Li W. 1991. Applied nonlinear control. New Jersey: PrenticeHall.

Soechting JF, Flanders M. 1997. Evaluating an integrated musculoskeletal model of the human arm. J Biomech Eng, 119:93-102.

Uno Y, Kawato M, Suzuki R. 1989. Formation and control of optimal trajectory in human multijoint arm movement: minimum torquechange model. Biol Cybern, 61:89-101.

Vincent TL, Grantham WJ. 1997. Nonlinear and optimal control systems. New York: J Wiley.

Zajac FE, Gordon ME. 1989. Determining muscle's force and action in multi-articular movement. Exerc Sport Sci Rev, 17:187-230. 

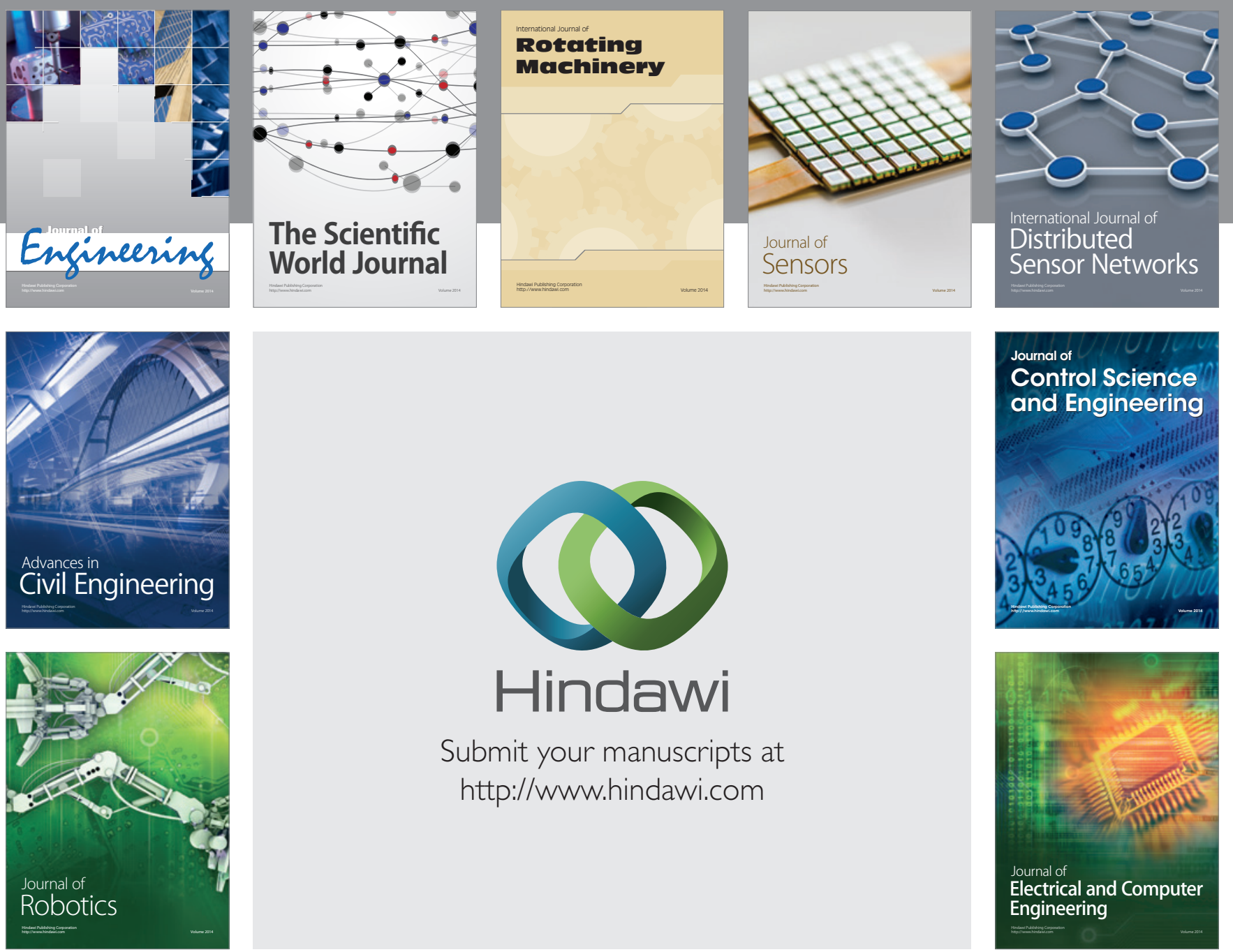

Submit your manuscripts at

http://www.hindawi.com
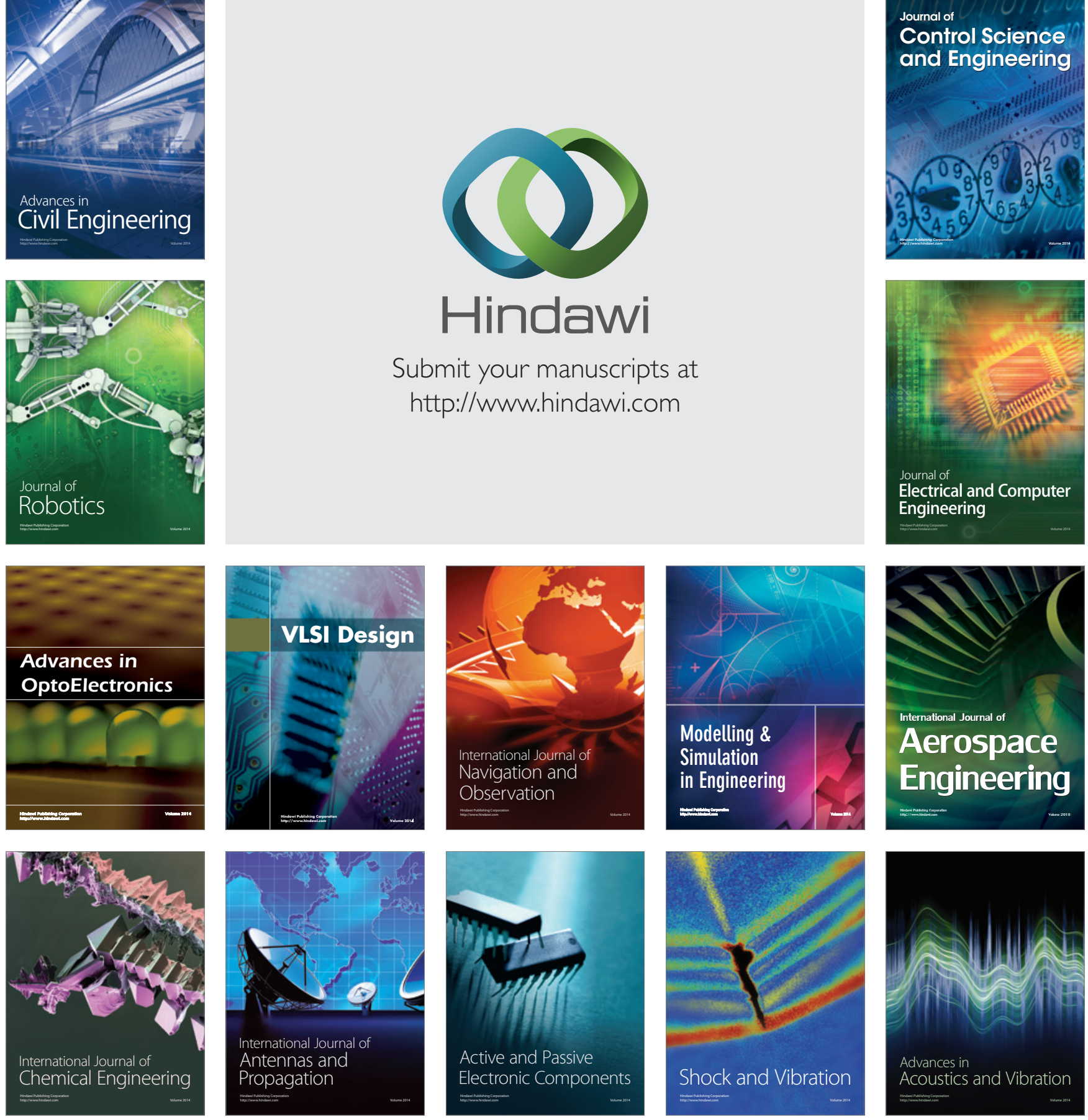